\title{
Pegademase bovine (PEG-ADA) for the treatment of infants and children with severe combined immunodeficiency (SCID)
}

This article was published in the following Dove Press journal:

Biologics: Targets \& Therapy

18 June 2009

Number of times this article has been viewed

\author{
Claire Booth ${ }^{1,2}$ \\ H Bobby Gaspar ${ }^{1,2}$ \\ 'Centre for Immunodeficiency, \\ Molecular Immunology Unit, \\ UCL Institute of Child Health, \\ London, UK; ${ }^{2}$ Dept of Clinical \\ Immunology, Great Ormond Street \\ Hospital NHS Trust, London, UK
}

Correspondence: HB Gaspar Molecular Immunology Unit, UCL Institute of Child Health, 30 Guilford Street, London WCIN IEH

Tel +4420790523I9

Fax +44 2079052810

Email h.gaspar@ich.ucl.ac.uk

\begin{abstract}
Adenosine deaminase deficiency (ADA) is a rare, inherited disorder of purine metabolism characterized by immunodeficiency, failure to thrive and metabolic abnormalities. A lack of the enzyme ADA allows accumulation of toxic metabolites causing defects of both cell mediated and humoral immunity leading to ADA severe combined immune deficiency (SCID), a condition that can be fatal in early infancy if left untreated. Hematopoietic stem cell transplant is curative but is dependent on a good donor match. Other therapeutic options include enzyme replacement therapy (ERT) with pegademase bovine (PEG-ADA) and more recently gene therapy. PEG-ADA has been used in over 150 patients worldwide and has allowed stabilization of patients awaiting more definitive treatment with hematopoietic stem cell transplant. It affords both metabolic detoxification and protective immune function with patients remaining clinically well, but immune reconstitution is often suboptimal and may not be long lived. We discuss the pharmacokinetics, immune reconstitution, effects on systemic disease and side effects of treatment with PEG-ADA. We also review the long-term outcome of patients receiving ERT and discuss the role of PEG-ADA in the management of infants and children with ADA-SCID, alongside other therapeutic options.
\end{abstract}

Keywords: adenosine deaminase deficiency, PEG-ADA, enzyme replacement therapy, severe combined immune deficiency (SCID)

\section{Introduction}

Adenosine deaminase (ADA) deficiency is a rare inherited disorder of purine metabolism characterized by severe and recurrent infection, failure to thrive and metabolic abnormalities. Absence of the enzyme ADA allows accumulation of toxic metabolites resulting in a complete or partial deficiency of both cell-mediated and humoral immunity. ADA severe combined immune deficiency (SCID) is fatal within the first months of life if left untreated. Hematopoietic stem cell transplant is curative but dependent on a good donor match. Other therapeutic options include enzyme replacement therapy (ERT) with pegademase bovine (hereon referred to as PEG-ADA) and more recently, gene therapy. PEG-ADA was first used to treat ADA deficiency in 1986 and to date more than 150 patients worldwide have received this treatment. It is well tolerated and can restore immune function to protective levels but long-term follow up suggests that immune recovery is incomplete. The precise role of PEGADA alongside other treatment options is still to be determined but to date it has allowed stabilization of patients awaiting more definitive treatment and clinical well-being in individuals treated for more prolonged periods. 


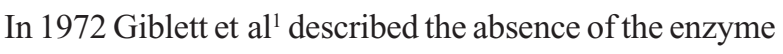
ADA in erythrocytes in two patients with combined immune deficiency. Subsequently, with greater awareness, more patients with similar findings were reported and the clinical and immunological phenotype of ADA deficiency was established. Patients can be divided into four phenotypes; ADA SCID, delayed onset ADA deficiency, late onset ADA deficiency and partial ADA deficiency. The largest number of patients ( $\sim 85 \%$ to $90 \%)$ present as ADA SCID and represent the most severe form of immunodeficiency; delayed onset presentation accounts for $10 \%$ to $15 \%$ of cases and late (or adult) onset deficiency has been identified in a handful of individuals. Partial deficiency can be an incidental finding in a healthy individual where immune function is normal but ADA expression is abnormal in erythrocytes and is again extremely rare. The incidence of ADA deficiency is estimated to be between 1 in 200,000 and 1 in 1,000,000 births $^{2}$ and accounts for around $15 \%$ to $20 \%$ of all SCID cases, although the true incidence of the disease is undetermined and may be higher in specific ethnic populations worldwide.

\section{Metabolic abnormalities}

ADA is a predominantly cytoplasmic enzyme found in all tissues of the body where it plays an important role in the recycling of adenosine after DNA breakdown and specifically catalyzes the deamination of deoxyadenosine (dAdo) and adenosine (Ado) to deoxyinosine and inosine respectively. ${ }^{3}$ An extracellular form of the enzyme (ecto-ADA) is found on the surface of many cell types where it binds the cell surface protein CD26 and specific adenosine receptors. Although ubiquitously expressed in keeping with its role as a 'housekeeping' enzyme, ADA expression levels are highest in the thymus and in lymphocytes where levels are 800- to 1000 -fold higher than in erythrocytes. ${ }^{4}$ High levels are also found in the foregut and brain. The lack of ADA results in a number of metabolic derangements which ultimately lead to the accumulation of toxic substrates in both intra- and extracellular compartments. The high levels of ADA activity in the thymus may be a direct consequence of the high level of cell turnover during thymic lymphocyte selection and the requirement for rapid and extensive DNA synthesis. Despite its importance for many organ systems, it is clear that the lack of ADA has its most profound manifestations in the immune system and in lymphocyte development and function.

Lack of ADA activity leads to the accumulation of Ado and dAdo. Cells compensate for rising levels of dAdo by converting intracellular dAdo to deoxyadenosine triphosphate (dATP) through the action of deoxycytidine kinase
(dCyd kinase). Increased dAdo levels inactivate the enzyme S-adenosyl homocysteine hydrolase (SAHH) leading to the accumulation of S-adenosyl homocysteine (AdoHcy) which is a potent inhibitor of transmethylation reactions. SAHH activity in patients with ADA deficiency is usually $<5 \%$ of normal levels. ${ }^{2}$

Elevated dATP levels have several toxic effects which may all play a role in lymphotoxicity and dATP levels have been shown to correlate with clinical severity. It has been shown in both ADA-deficient patients and murine models that dATP pool expansion leads to inhibition of ribonucelotide reductase required for DNA replication and repair ${ }^{5}$ and in induction of apoptosis in immature thymocytes. ${ }^{2}$ Because cells with a high turnover produce more dATP, apoptosis, which is the fate of most lymphocytes entering the thymus, also increases the dATP pool within the thymus. Limited antigen receptor diversity has been demonstrated through interference of dATP with terminal deoxynycleotidyl transferase $(\mathrm{TdT})^{6}$ and defective signaling pathways may also impair lymphocyte function. ${ }^{2}$ Apoptosis in thymocytes is also induced by accumulated extracellular adenosine acting through $\mathrm{A}(2 \mathrm{~A})$ adenosine receptors.

\section{Presentation}

Children with ADA SCID present within the first months of life with failure to thrive, life threatening infections and profound lymphopenia. Interstitial pneumonitis is a common feature and may be caused by a viral pathogen or commonly Pneumocystis jiroveci but may also be an intrinsic manifestation of ADA deficiency. Pulmonary alveolar proteinosis (PAP) has been identified radiologically and histologically in ADA-deficient patients and is reversible upon treatment (Grunebaum et al, Abstract at ESID 2008). Results from broncheoalveolar lavage (BAL) undertaken in ADA SCID patients with clinical and radiological manifestations of pneumonitis revealed a high percentage of children in whom no pathogen was identified (Gaspar, unpublished data) again suggesting that pulmonary damage in ADA-deficient patients is an intrinsic consequence of the disease. Infants may also suffer from opportunistic infections in other organ systems, diarrhoea and candidiasis. Clinical examination reveals physical signs usually related to infection and poor growth and the absence of lymph nodes and tonsillar tissue due to the lack of secondary lymphoid organ development. The thymic shadow may be absent or reduced on chest radiography and blood tests reveal a profound lymphopenia with greatly reduced numbers of T, B and NK cells leading to T-B-NK- SCID phenotype. Functional lymphocyte studies unsurprisingly show absent 
proliferation in response to mitogen stimulation and lack of immunoglobulin production.

Immunodeficiency is the most profound, and in the majority of cases first manifestation of ADA deficiency but the ubiquitous expression of ADA results in abnormalities in other organ systems. Since untreated children invariably have a short life expectancy, non-immunological deficits have usually been recognized once the immunodeficiency has been corrected by bone marrow transplantation. Abnormal neurological findings, without evidence of central nervous system infection, such as developmental delay, abnormal muscle tone and sensorineural deafness are well described. High levels of ADA expression have been reported in brain tissue ${ }^{7}$ and it is possible that high levels of dATP are neurotoxic. Cognitive function is adversely affected by the severity of metabolic derangement at diagnosis and a significant inverse correlation has been shown between dATP levels at diagnosis and IQ score. ${ }^{8}$ Significant behavioral abnormalities are also seen in these patients which is not related to the process of transplantation as demonstrated by a case-matched study. ${ }^{8}$ Post mortem findings in eight children with ADA deficiency revealed non-lymphoid multisystem pathologic changes, mainly renal and skeletal. ${ }^{9}$ Autoimmune phenomena such as idiopathic thrombocytopenia and hemolytic anemia are well described. ${ }^{10}$ Other rare presenting features include insulin dependent diabetes mellitus with atopic dermatitis ${ }^{11}$ and hepatitis. ${ }^{12}$

Delayed onset ADA deficiency is the term applied to patients who present after the first year of life and is most likely related to the specific ADA mutation which in such cases results in some residual ADA activity and less severe dATP expansion and consequently less profound lymphopenia and immune function. Such patients present with recurrent infection and lymphopenia but rarely infection with opportunistic pathogens. Immune dysregulation, autoimmune and allergic phenomena and cytopenias may also be a feature of this phenotype. A history of recurrent otitis media, upper respiratory tract infection or pneumonia is more commonly seen and often these children do not require hospitalization initially. Misdiagnosis with other conditions such as allergy (due to raised eosinophil counts) or antibody deficiency is not uncommon.

Early diagnosis is essential to prevent the complications of chronic infection and allow normal growth. Lymphocyte subsets and functional studies, proliferation studies and immunoglobulin levels are important but diagnosis rests on the demonstration of absent or very low plasma or erythrocyte ADA activity levels. Raised dATP levels and reduced SAHH activity in erythrocytes also confirm a diagnosis.
Genetic testing using mutation analysis is available and over 70 different mutations (mostly missense mutations) have been identified ${ }^{13}$ involving the ADA gene on chromosome 20q13.11. ${ }^{14}$ Prenatal diagnosis may be performed by either mutation analysis or by measurement of enzymatic activity in trophoblasts from chorionic villus sampling or amniocentesis. ${ }^{15}$

\section{Management options}

Currently there are three management options to treat ADA deficiency: hematopoietic stem cell transplant (HSCT), ERT and gene therapy. HSCT is a highly successful and curative procedure if a matched related donor is available with survival rates of $\sim 90 \%$ but this rate falls to nearer $50 \%$ survival if a matched unrelated donor or haploidentical transplant is undertaken. ${ }^{16}$ Matched family donor transplants can be successfully undertaken without the need for prior cytoreductive chemotherapy but transplants from other donor sources require the use of a chemotherapy regimen. Immune reconstitution post transplant confers adequate immunity in terms of lymphocyte numbers and function and antibody production. Metabolic correction is also seen and although dATP levels are reduced they remain higher than in a normal individual (and indeed higher than in patients on ERT). HSCT is the treatment option of choice if a well matched family donor can be found. However, HSCT does not correct the non-immunological manifestations of ADA deficiency which again suggests that ADA deficiency is a systemic disease.

More recently somatic gene therapy for the correction of ADA deficiency has evolved, offering patients another treatment option. The procedure involves gammaretroviral mediated introduction of the human ADA gene into autologous bone marrow progenitors and subsequent infusion of cells back to the patient following mild nonmyeloablative chemotherapy. Preliminary results from clinical trials are encouraging with the majority of patients experiencing immune reconstitution and metabolic detoxification with stable engraftment of transduced hematopoietic cells. ${ }^{17,18}$ Importantly there has been little toxicity from the procedure and to date all patients have survived. Immune reconstitution appears to be similar to that seen post transplant. Over 25 patients have now been treated at four centers worldwide, with the most extensive experience in Milan and London. The protocols differ between the different sites with respect to the specific retroviral constructs used for gene transfer and the conditioning protocols used. Although the majority of patients have shown improved immune recovery after 
gene therapy, the level of reconstitution appears variable and seems to be determined by a number of factors including, the number of gene corrected progenitor cells re-infused into the patients, the ability to harvest sufficient stem cells and the degree of thymic reserve at the time of gene therapy. The kinetics of immune recovery are also delayed when compared to the $\mathrm{T}$ cell reconstitution following gene therapy for the X-linked form of SCID. However a number of patients show long-term ( $>5$ years) complete $\mathrm{T}$ cell and humoral recovery and have been able to discontinue all prophylactic medication and immunoglobulin replacement therapy. These pioneering studies suggest that gene therapy can at least in principle provide effective immune and metabolic recovery. The success of gene therapy is tempered by the potential for insertional mutagenesis and leukemic transformation as a result of using gammaretroviral vectors. ${ }^{19,20}$ These complications have been seen in trials of gene therapy for other immunodeficiencies but not so far in ADA gene therapy studies. Gene therapy remains an option if well matched HSCT donor is not available and is only possible through recruitment at specialized academic centers.

\section{Red cell transfusions}

Originally enzyme replacement was delivered through erythrocyte transfusions after work by Polmar et al demonstrated addition, in vitro, of calf-intestinal ADA or human erythrocyte ADA to ADA deficient patients lymphocytes restored proliferation after mitogen stimulation. ${ }^{21}$ Several patients were treated with regular transfusions of frozen, irradiated red blood cells every 2 to 4 weeks. Initial reports were promising indicating an improvement not only in clinical status but also in immunological function. Children remained free of infection for up to 2 years and showed an improvement in lymphocyte numbers and function. ${ }^{22,23}$ Metabolic derangements were also seen to normalize with an increase in ADA activity, reduction in adenosine and deoxyadenosine levels and a decrease in erythrocyte dATP. ${ }^{23-25}$ However, these improvements proved to be transient and dependent on regular transfusions. ${ }^{25}$ Long-term transfusion therapy carries with it certain risks including iron overload, exposure to potential blood borne viral pathogens and the trauma of repeated cannulation and time in hospital. These are significant risks in immunodeficient children who already spend much time in a hospital environment. Bax et $\mathrm{al}^{26}$ reported a patient diagnosed in her mid-thirties with ADA deficiency who was treated for 9 years with autologous erythrocyte encapsulated ADA infusions (after one year of combined PEG-ADA injections). The patient was not a candidate for
HSCT but this treatment proved metabolically and clinically effective in this case.

Later studies did not show such a positive outcome with regular red blood cell transfusions and in fact, demonstrated insufficient metabolic correction to allow for immune recovery, although clinical status did often improve. ${ }^{25,27}$ After the introduction of PEG-ADA a comparative study proved PEG-ADA to be a superior form of enzyme replacement and red cell transfusion was rendered obsolete. ${ }^{27}$

\section{Enzyme replacement therapy}

ERT with PEG-ADA (Adagen ${ }^{\circledR}$; Enzon Inc; obtained outside USA through Orphan Europe) for the treatment of ADA deficiency has been available for almost 20 years and has been designated an orphan drug. The use of PEG-ADA provides another treatment modality for ADA SCID, however unlike HSCT or gene therapy it is not a curative therapy but requires regular intramuscular administration. Nevertheless, the effectiveness of PEG-ADA in correcting metabolic and immunological parameters and more importantly in promoting clinical well-being in patients, makes it an important option in the care of patients. The question remains as to the timing and specific role of the three different treatment modalities in the treatment of ADA SCID patients and is the subject of more detailed discussion below.

\section{PEGylation}

PEGylation, a process pioneered in the 1970 s, involves the covalent attachment of numerous strands of monomethoxypolyethylene glycol (PEG) to a molecule, for example an enzyme or protein. PEG itself is neither toxic nor immunogenic. The attachment of PEG to ADA through lysine residues confers several therapeutically beneficial properties to ADA through alteration of its physical and chemical properties, mainly due to an increase in molecular weight. ${ }^{28,29}$ The circulating life of the compound is prolonged from minutes to days as clearance from the circulation is inhibited. Molecules are protected somewhat from cellular uptake, proteolytic attack, renal clearance, antibody binding and antigen presentation. PEGylation also reduces the immunogenicity of a protein which again helps to extend its circulating life. ${ }^{28,29}$ In theory, if a drug or protein remains in the circulation for a longer period then the frequency of administration could be reduced thus reducing cost and the number of injections a patient requires. In 1986, Hershfield et al first used ADA derived from bovine intestine conjugated to PEG to treat patients with ADA deficiency. ${ }^{30}$

Other PEGylated therapies currently used in clinical practice include PEGASYS ${ }^{\circledR}$ (Hoffman-La Roche) 
and PEG-Intron ${ }^{\circledR}$ (Schering-Plough/Enzon) which are PEGylated alpha-interferons used to treat chronic hepatitis $\mathrm{B}$ and $\mathrm{C}$ and PEGylated L-asparaginase (Oncaspar ${ }^{\circledR}$; Enzon) used for the treatment of acute lymphoblastic leukemia.

The emergence of new variant Creutzfeldt-Jakob disease (CJD) has led to concerns over the use of bovine ADA as the source of enzyme for PEG-ADA. Bovine ADA is now extracted from bovine herds in countries where nvCJD has not been identified. There has also been much speculation over whether a human recombinant form of ADA may be developed.

\section{Kinetics and dosage}

PEG-ADA is administered by intramuscular injection once or twice weekly. Plasma levels of ADA activity peak 24 to 48 hours after injection and the half life varies from 3 to more than 6 days. The elimination and pharmacokinetics of PEG-ADA are variable within the same patient and monitoring of plasma ADA activity helps to guide dosage and frequency of administration. Reports also suggest that clearance is enhanced in younger infants and more severely ill children. ${ }^{2,31}$ Cellular uptake of PEG-ADA is not significant but maintaining plasma ADA levels $>100$-fold normal levels leads to a reduction in extracellular Ado and dAdo levels and subsequent normalization of intracellular levels ${ }^{16}$ through maintenance of equilibrium between intra- and extracellular compartments.

Since the first patients were treated, the dosing regime has evolved and it is now advised that children should start at a dose of $60 \mathrm{U} / \mathrm{kg} /$ week with biweekly injections until metabolic correction is established (between 1 and 3 months). Once patients show clinical improvement and biochemical stabilization they can be maintained on a dose of $30 \mathrm{U} / \mathrm{kg} /$ week in a single weekly injection, ${ }^{32,33}$ unpub. It is important when monitoring patients to assess their immune function as well as metabolic parameters and this should be taken into account when considering altering the dose of PEG-ADA.

Dosage should be adjusted according to trough levels of plasma ADA activity. Erythrocyte dATP levels can also be measured and used to guide treatment. Initial trough plasma ADA levels (prior to injection) should be maintained at $50-150 \mu \mathrm{mol} / \mathrm{h} / \mathrm{mL}$ (normal range $<0.4 \mu \mathrm{mol} / \mathrm{h} / \mathrm{mL}$ ) which equates to approximately 4 to 10 times the normal erythrocyte $\mathrm{ADA}$ activity ${ }^{2}$ and is required for intial rapid detoxification. Once a maintenance dose of $30 \mathrm{U} / \mathrm{kg} /$ week is established, trough plasma ADA levels can be maintained at 25 to $60 \mu \mathrm{mol} / \mathrm{h} / \mathrm{mL}$. Erythrocyte dATP levels decrease significantly and are maintained at levels below that seen after HSCT. In addition, SAHH activity is seen to normalize.
It is recommended that trough plasma ADA activity levels are monitored every 1 to 2 weeks during the first 2 to 3 months of treatment, twice a month until 9 months of treatment and then monthly until 18 to 24 months on PEG-ADA. Once patients are established on an effective maintenance dose then plasma ADA levels can be measured every 2 to 4 months unless there is a change in clinical status.

\section{Immune reconstitution}

Immune reconstitution following treatment with PEG-ADA has to date not been followed in a prospective manner and therefore formal data are not available. The reasons for this are varied but most probably due to the rarity of the condition and the fact that any one center treats only a handful of patients. The data cited below are therefore taken from retrospective and single-center studies which stretch back over the last 20 years. Overall, it is clear that immune recovery is very variable, the reasons for which may be associated with the underlying clinical condition of the child, the age at which treatment is started and also the level of residual thymic activity at the time of PEG-ADA initiation. Up to $20 \%$ of patients receiving therapy show no response. ${ }^{2,33}$ In the majority of cases, full immune recovery is seen in the short term but is followed by waning $\mathrm{T}$ cell numbers. ${ }^{10} \mathrm{In}$ terms of humoral immunity, continued immunoglobulin replacement is required in up to $50 \%$ of those treated with long-term PEG-ADA. ${ }^{16}$ It is also important to document that, from the retrospective reports available and from communication with physicians treating patients, despite low $\mathrm{T}$ cell reconstitution and lack of humoral recovery, clinical well being is maintained and children who have been treated for many years remain clinically well, free of infection, with normal growth parameters.

Immune recovery is evidenced initially by a rise in B cell numbers within a few weeks of starting treatment and is then followed by a rise in $\mathrm{T}$ cell count which may take a number of months to occur. Symptoms of immune dysregulation such as hemolytic anemia and immune thrombocytopenia can be seen during this period and may be related to dysregulated cellular and humoral recovery. Immature $\mathrm{T}$ lymphocytes appear first and account for the rise in T cell numbers. Proliferative responses can be detected once mature $\mathrm{T}$ lymphocytes develop demonstrating that ADA-deficient thymic progenitors are able to mature into functional $\mathrm{T}$ lymphocytes in the presence of metabolic correction. ${ }^{31}$ Initially these responses are IL-2 dependent and later become IL-2 independent. ${ }^{31}$ Long-term outcome data suggest that mitogen responses often fluctuate within the same patient over time ${ }^{32}$ with 
normal responses evident in only about $50 \%$ of patients. Specific antigen responses return in the majority of children over a similar time frame to that seen post HSCT.

Antibody responses to immunization with bacteriophage $\Phi \times 174$ are useful as this is an antigen not present in replacement immunoglobulin. ${ }^{34}$ Serum immunoglobulin levels generally rise after a few months of treatment but this is not uniform and as mentioned previously, up to half of children receiving $\mathrm{PEG}$-ADA require ongoing immunoglobulin replacement. NK cell activity has been documented in one patient as returning after 5 months. ${ }^{31}$

Initial reports of short-term follow up of patients treated with PEG-ADA were promising, demonstrating partial but protective immune recovery and clinical improvement with a reduction in the number and severity of infections. However, even shortly after clinical trials began it was evident that immune reconstitution faltered after a few years of treatment despite maintaining stable plasma ADA levels conducive with immune recovery. ${ }^{32}$

Deaths have been reported within the first 6 months of treatment (mainly in the first month) but it is likely that this represented mortality due to infection at diagnosis and was not related to PEG-ADA treatment.

Around $30 \%$ of those treated with PEG-ADA have a delayed onset phenoytype. ${ }^{16}$ These patients are particular, often suffering the effects of chronic infection coupled with some residual immune function which can complicate engraftment following HSCT. One case report describes a 14-month-old girl with delayed onset ADA deficiency who responded well to PEG-ADA therapy showing almost full immune recovery but went on to develop inhibitory IgG anti-ADA antibodies which resulted in reaccumulation of toxic metabolites and subsequent reversal of immune reconstitution. ${ }^{4}$ Older patients have been treated successfully and are able to lead a normal life, including schooling. ${ }^{32,35}$

\section{Long-term outcome in patients treated with PEG-ADA}

Data on long-term outcome in patients with ADA deficiency treated with PEG-ADA has been published by several groups. ${ }^{16,36,37}$ Chan et $\mathrm{al}^{37}$ published a retrospective review of five patients treated in North America with follow up data from 1995 to 2002 . The patients were mostly typical ADA-SCID patients with early presentation (one late onset patient) who had received PEG-ADA for 5 to 15 years. Their clinical course over this time was good with little in the way of recurrent or severe infections, although any immune reconstitution associated with treatment declined over time.
The majority of children also received other forms of treatment including HSCT and gene therapy. Absolute lymphocyte counts (ALC) remained below the normal range despite improving from baseline. ALC peaked between 1 to 3 years on PEG-ADA at 250 to 1480 cells $/ \mathrm{mm}^{3}$ but fell after 5 to 12 years of therapy to 12 to 500 cells $/ \mathrm{mm}^{3}$. It was noted that the changing CD4:CD8 ratio seen with increasing age was maintained. B cell numbers showed an improvement that was not sustained, peaking at 60 to 1270 cells $/ \mathrm{mm}^{3}$ then falling to subnormal range. NK cells were also monitored in most patients and reached normal numbers (120 to 2400 cells $/ \mathrm{mm}^{3}$ ) before falling to 30 to 230 cells $/ \mathrm{mm}^{3}$. Proliferative responses normalized in some patients after an average of 4 months of treatment before declining over time. Immunglobulin production was difficult to assess as patients received supplemental immunoglobulin. It was postulated that a reversal of immune recovery may be related to a reduction in thymopoeisis which naturally occurs as a child grows older. Low levels of ADA in the thymus are known to be toxic to lymphocyte precursors entering the thymus ${ }^{38}$ and it may be that irreversible damage is already present in the thymus before any treatment is started (as toxicity is present in utero, and damage may begin before birth), and severely affects the potential for lymphocyte development and proliferation.

Malacarne et $\mathrm{al}^{36}$ explored thymic output and immune reconstitution in five patients (ages 5 to 9 years) with ADA SCID treated with PEG-ADA for 5 to 8 years. B cell and $\mathrm{T}$ cell numbers again increased 5 to 14 months following initiation of treatment but remained low in comparison to normal controls. Patient responses to PHA (mitogen) stimulation increased but were variable even within the same patient. Four of the five patients developed specific antibodies after immunization with tetanus and showed an increase in serum immunoglobulin levels. Normal T cell development and thymic output were measured through $\mathrm{T}$ cell receptor excision circles (TRECS) and these proved to be consistently low compared with healthy age matched controls again suggesting a compromise in thymic function. Accelerated apoptosis was also demonstrated in patients $\mathrm{T}$ lymphocytes. B cell repertoire was examined and found to be restricted but it is not clear if this relates to an intrinsic B cell problem or secondary to defects in the T cell compartment. Patients remained clinically well on long-term therapy with PEG-ADA.

A European survey of patients receiving PEG-ADA therapy was undertaken in 2005 (involving the ESID and EBMT Inborn Errors Working Parties) and preliminary data were published in 2007. ${ }^{16}$ Data gathered from 42 patients based in several European centers showed that PEG-ADA was 
started in the first 6 months of life in over half of the patients $(n=27)$. Two thirds of patients received PEG-ADA only and the remainder progressed to HSCT or gene therapy. Four reported deaths were infection related (CMV viremia, RSV infection, encephalitis and pneumonitis) and were unlikely to be related to PEG-ADA. As previously reported, immune recovery was variable with $\mathrm{T}$ lymphocyte numbers below the normal range after one year of treatment (mean CD3+ count: 460 cells $/ \mathrm{mm}^{3}$ ). Immunoglobulin levels improved to the extent that $40 \%$ of patients received immunoglobulin replacement after one year. Overall survival in this cohort was $85 \%$ for children who received PEG-ADA alone $(n=26)$. Survival for those who went onto receive HSCT was 70\% and $100 \%$ for those progressing to gene therapy.

At present the long-term consequences of PEG-ADA therapy are unknown but it is clear that immune recovery especially in the $\mathrm{T}$ cell compartment is below normal levels and in some patients runs at levels that are of concern for opportunistic infection. The reasons for this have not been determined but reduced thymic function either at the onset of therapy or over the course of therapy may play an important role. The majority of patients have remained clinically well without experiencing major infective problems but a number of case reports suggest that in certain patients, ongoing low $\mathrm{T}$ cell numbers has led to significant problems. One boy developed Hodgkin's lymphoma after 13 years of treatment, ${ }^{10}$ another developed Burkitt's lymphoma again after 13 years of treatment ${ }^{39}$ and another child developed EBV positive malignant brain lymphoma after 10 years of treatment. ${ }^{40}$ It is likely that such lymphoproliferative diseases arise due to reduced immune surveillance. Further data gathering is necessary to determine overall outcome of patients on long-term therapy.

\section{Effects of PEG-ADA on systemic disease}

The effects of PEG-ADA on the metabolic disturbances and immune reconstitution seen in ADA deficiency have been well described but its effect on systemic manifestations remain unclear. In a number of major centers, children with ADA deficiency proceed to HSCT when an appropriate donor becomes available and PEG-ADA treatment is discontinued and therefore long-term systemic outcome data are lacking. In those patients that have remained on long-term ERT, there are no formal data on the outcome of systemic pathology. Certainly, the cognitive and behavioral abnormalities and sensorineural deafness described in ADA-deficient patients are not affected by metabolic correction and persist after transplantation and these are now well documented but no such data exists for patients on PEG-ADA. This is perhaps because no individual center has a sufficiently large cohort on long-term enzyme replacement to perform such a study and because such studies especially on behavioral function are difficult to perform across a number of different countries and continents.

Some information may be inferred from murine studies. ADA-deficient mice exhibit severe pulmonary insufficiency in addition to combined immunodeficiency, renal abnormalities, hepatocellular degeneration and failure to thrive. ${ }^{12,41}$ Mice die before 3 weeks of age from respiratory disease. Pulmonary pathology appears to be a primary consequence of ADA deficiency and not due to infectious damage ${ }^{12}$ and has been well characterized in the mouse model. Abnormal adenosine signaling leads to a developmental defect in alveogenesis resulting in enlarged alveolar airways and severe lung inflammation. This process appears to be separate to the immune consequences of ADA deficiency which are due to cytotoxicity of deoxyadenosine and metabolic derangements. Blackburn et $\mathrm{a}^{42}$ demonstrated that a relatively 'low dose' of PEG-ADA was sufficient to prevent the pulmonary phenotype but resulted in no change in immunodeficiency. Reduced levels of dATP were noted in the lungs and erythrocytes with 'low dose' replacement with an improvement in the clinical phenotype and resulted in significantly improved survival of treated mice. When mice received 'high dose' enzyme replacement a reduction in the metabolic disturbance in the thymus and spleen was seen and immune function improved.

There is a case report of a neonate presenting with hepatitis and prolonged hyperbilirubinemia who subsequently developed immune deficiency and was diagnosed with ADA deficiency. ${ }^{12}$ After commencing ERT, liver dysfunction improved and was followed shortly after by improvement in lymphocyte count. These data suggest that PEG-ADA, through its ability to rapidly detoxify the metabolic consequences of ADA deficiency, is able to reverse a number of the clinical pathologies.

\section{Side effects}

To date, no toxic or hypersensitivity reactions have been reported with Adagen ${ }^{\circledR}$. Headache and pain at the injection site were reported adverse effects during the clinical trial. After approval by the FDA several other side effects have been reported including hemolytic anemia, autoimmune hemolytic anemia, thrombocytopenia and erythema or urticaria around the injection site.

The main side effect associated with the use of PEG-ADA which will be discussed in more detail is the development of anti-ADA antibody. Transient immune 
dysregulation can occur during the first few months of treatment as may occur post transplant. One patient described developed thrombocytopenia that responded to treatment with intravenous immunoglobulin and several others developed a more persistent hemolytic anemia, in some cases associated with intercurrent viral infection or sepsis., ${ }^{2,10}$ Transient thrombocytosis (an increased platelet count) has been reported by several groups but this has not resulted in any clinical sequelae such as thrombosis. ${ }^{16,43}$ Having reviewed data from another two patients being treated with PEGADA at one center, a rise in platelet count was noted after PEG-ADA therapy was commenced, but this was within the normal range for the majority of treatment. The mechanism by which this may occur or be significant is not clear but It was speculated that reconstitution of the immune system in response to infection may play a role.

The development of specific IgG antibody to bovine peptide epitopes of PEG-ADA has been reported by several groups and often coincides with an improvement in humoral immunity., ${ }^{4,16,33,44}$ There are no reports of antibody formation to PEG itself. It can be detected by ELISA in up to $80 \%$ of patients on long-term PEG-ADA therapy but is clinically insignificant in most. However, in around $10 \%$ of those treated an inhibitory antibody is produced leading to enhanced clearance of PEG-ADA and subsequently a decline in metabolic parameters and immune function. ${ }^{4,33,44}$ Patients with delayed or late onset phenotype seem particularly susceptible to the development of neutralizing antibody. ${ }^{10}$ Development of anti-ADA antibody should be suspected if plasma ADA activity consistently falls below $10 \mu \mathrm{mol} / \mathrm{h} / \mathrm{mL}$ and an ELISA can be performed to confirm its presence.

Chaffee et $\mathrm{al}^{44}$ describe the development of significant anti-ADA antibody levels ( $>2$ SD mean control group) in 10 out of 17 patients between 3 and 8 months of treatment. Stable plasma ADA activity was maintained in eight patients but in two patients was reduced to such a level as to have an effect on clinical status and require modification in their management. One patient responded to twice weekly injections rather than weekly. For the other patient PEG-ADA was withheld and they were treated with intravenous immunoglobulin and prednisolone to induce tolerance before recommencing on PEG-ADA. Both human and bovine derived ADA were inhibited by these two patients' antibodies suggesting that the antibody recognized a conserved region of the enzyme.

\section{Costs of PEG-ADA}

As with many orphan drugs, the cost of PEG-ADA usage is high and may prohibit its use in resource poor countries or where insurance cover is not readily available. However, the cost appears to be in keeping with enzyme replacement for other metabolic conditions and reflects the costs of development and production for use in a limited patient population.

\section{The role of PEG-ADA}

Enzyme replacement therapy with pegylated ADA provides metabolic correction and improvement in immune function and clinical parameters. However it is not curative and longterm immune recovery is of concern. The role of PEG-ADA has to be set alongside the other treatment options of HSCT and gene therapy. At present there is limited formal data on the outcome following HSCT after different donor transplants and gene therapy trials are confined to $\sim 20$ to 25 patients worldwide. Therefore, arriving at any didactic management schema is difficult and is often subject to personal preference, experience and availability.

Despite these caveats, the consensus suggests that where a matched family donor is available the overall success rates are very high ( $>90 \%$ survival with effective immune reconstitution), and thus HSCT is the treatment of choice. Even in this donor situation, PEG-ADA may be important in stabilizing the child through metabolic detoxification prior to transplant. If a matched family donor is unavailable, then most centers would start PEG-ADA while initiating an unrelated donor search in order to stabilize the child and to improve metabolic and immune recovery. Following this, there are little formal data to guide how best to proceed since the options now include continuation of PEG-ADA, unrelated donor transplant or gene therapy. This decision is most likely to be dictated by the experience of individual physicians and the treatment options available at specific institutions. Equally the time for which PEG-ADA should be continued is not clear given the poor long-term immune recovery seen in patients who nevertheless remain clinically stable. Again, this may be resolved once data from large numbers of patients on long-term PEG-ADA therapy are available.

PEG-ADA was given as an adjunctive therapy in previous gene therapy trials ${ }^{45,46}$ but it is recognized that enzyme replacement may blunt the survival advantage of gene corrected cells and therefore patients have been maintained on PEG-ADA up until the time of gene therapy, at which point it has been discontinued. However, the use of PEG-ADA therapy may be important prior to gene therapy in the maintenance of a healthy hematopoietic stem cell pool from which sufficient cells can be harvested for the transduction procedure (Gaspar, unpublished data). Patients who are 
on ERT discontinue treatment shortly prior to a transplant procedure. There are as yet no reports of patients who have successfully undergone transplant or gene therapy restarting PEG-ADA even if there are ongoing non-immunological abnormalities.

\section{Summary}

The development and use of PEG-ADA has provided an important alternative option for treatment of patients with ADA deficiency. The rapid metabolic detoxification afforded by high level enzyme replacement allows clinical stabilization of patients and provides longer term treatment options when no suitable donor is available. The long-term immune recovery on PEG-ADA appears to be suboptimal although clinical well-being is maintained in the majority of patients. Further long-term data gathered from patients who have undergone the different treatment options available for ADA deficiency will allow more evidence-based recommendations for the role of PEG-ADA in disease management.

\section{References}

1. Giblett ER, Anderson JE, Cohen F, Pollara B, Meuwissen HJ. Adenosine-deaminase deficiency in two patients with severely impaired cellular immunity. Lancet. 1972;2(7786):1067-1069.

2. Hershfield M, Mitchell BS. Immunodeficiency diseases caused by adenosine deaminase deficiency and purine nucleoside phosphorylase deficiency. In: Scriver CR, Beaudet AL, Sly WS, Valle D, editors. The Metabolic and Molecular Basis of Inherited Disease. New York: McGraw-Hill; 2001. p. 2585-2625.

3. Hirschhorn R. Overview of biochemical abnormalities and molecular genetics of adenosine deaminase deficiency. Pediatr Res. 1993; 33(1 Suppl):S35-S41.

4. Lainka E, Hershfield MS, Santisteban I, et al. polyethylene glycolconjugated adenosine deaminase (ADA) therapy provides temporary immune reconstitution to a child with delayed-onset ADA deficiency. Clin Diagn Lab Immunol. 2005;12(7):861-866.

5. Lee N, Russell N, Ganeshaguru K, et al. Mechanisms of deoxyadenosine toxicity in human lymphoid cells in vitro: relevance to the therapeutic use of inhibitors of adenosine deaminase. $\mathrm{Br}$ J Haematol. 1984;56(1):107-119.

6. Gangi-Peterson L, Sorscher DH, Reynolds JW, Kepler TB, Mitchell BS. Nucleotide pool imbalance and adenosine deaminase deficiency induce alterations of $\mathrm{N}$-region insertions during $\mathrm{V}(\mathrm{D}) \mathrm{J}$ recombination. $J$ Clin Invest. 1999;103(6):833-841.

7. Hirschhorn R, Martiniuk F, Rosen FS. Adenosine deaminase activity in normal tissues and tissues from a child with severe combined immunodeficiency and adenosine deaminase deficiency. Clin Immunol Immunopathol. 1978;9(3):287-292.

8. Rogers MH, Lwin R, Fairbanks L, Gerritsen B, Gaspar HB. Cognitive and behavioral abnormalities in adenosine deaminase deficient severe combined immunodeficiency. J Pediatr. 2001;139(1):44-50.

9. Ratech H, Greco MA, Gallo G, et al. Pathologic findings in adenosine deaminase-deficient severe combined immunodeficiency. I. Kidney, adrenal, and chondro-osseous tissue alterations. Am J Pathol. 1985;120(1):157-169.

10. Hershfield MS. Combined immune deficiencies due to purine enzyme defects. In: Steim ER, Ochs HD, Winkelstein JA, editors. Immunologic Disorders in Infants and Children. Philadelphia: WB Saunders; 2004. p. $480-504$.
11. Notarangelo LD, Stoppoloni G, Toraldo R, et al. Insulin-dependent diabetes mellitus and severe atopic dermatitis in a child with adenosine deaminase deficiency. Eur J Pediatr. 1992;151(11):811-814.

12. Bollinger ME, rredondo-Vega FX, Santisteban I, et al. Brief report: hepatic dysfunction as a complication of adenosine deaminase deficiency. N Engl J Med. 1996;334(21):1367-1371.

13. Hirschhorn R. Immunodeficiency disease due to deficiency of adenosine deaminase. In: Puck JM, editor. Primary Immunodeficiency Diseases: A Molecular and Genetic Approach. New York, NY: Oxford; 1999. p. 121-139.

14. Petersen MB, Tranebjaerg L, Tommerup N, Nygaard P, Edwards H. New assignment of the adenosine deaminase gene locus to chromosome $20 \mathrm{q} 13 \times 11$ by study of a patient with interstitial deletion $20 \mathrm{q} . J$ Med Genet. 1987;24(2):93-96.

15. Dooley T, Fairbanks LD, Simmonds HA, et al. First trimester diagnosis of adenosine deaminase deficiency. Prenat Diagn. 1987;7(8):561-565.

16. Booth C, Hershfield M, Notarangelo L, et al. Management options for adenosine deaminase deficiency; proceedings of the EBMT satellite workshop (Hamburg, March 2006). Clin Immunol. 2007;123(2):139-147.

17. Aiuti A, Cattaneo F, Galimberti S, et al. Gene therapy for immunodeficiency due to adenosine deaminase deficiency. $N$ Engl J Med. 2009;360(5):447-458.

18. Gaspar HB, Bjorkegren E, Parsley K, et al. Successful reconstitution of immunity in ADA-SCID by stem cell gene therapy following cessation of PEG-ADA and use of mild preconditioning. Mol Ther. 2006;14(4):505-513.

19. Hacein-Bey-Abina S, Garrigue A, Wang GP, et al. Insertional oncogenesis in 4 patients after retrovirus-mediated gene therapy of SCID-X1. J Clin Invest. 2008;118(9):3132-3142.

20. Ott MG, Schmidt M, Schwarzwaelder K, et al. Correction of X-linked chronic granulomatous disease by gene therapy, augmented by insertional activation of MDS1-EVI1, PRDM16 or SETBP1. Nat Med. 2006;12(4):401-409.

21. Polmar SH, Wetzler EM, Stern RC, Hirschhorn R. Restoration of in-vitro lymphocyte responses with exogenous adenosine deaminase in a patient with severe combined immunodeficiency. Lancet. 1975;2(7938):743-746.

22. Polmar SH, Stern RC, Schwartz AL, et al. Enzyme replacement therapy for adenosine deaminase deficiency and severe combined immunodeficiency. N Engl J Med. 1976;295(24):1337-1343.

23. Dyminski JW, Daoud A, Lampkin BC, et al. Immunological and biochemical profiles in response to transfusion therapy in an adenosine deaminase-deficient patient with severe combined immunodeficiency disease. Clin Immunol Immunopathol. 1979;14(3):307-326.

24. Hirschhorn R, Paageorgiou PS, Kesarwala HH, Taft LT. Amerioration of neurologic abnormalities after "enzyme replacement" in adenosine deaminase deficiency. N Engl J Med. 1980;303(7):377-380.

25. Hutton JJ, Wiginton DA, Coleman MS, et al. Biochemical and functional abnormalities in lymphocytes from an adenosine deaminasedeficient patient during enzyme replacement therapy. $J$ Clin Invest. 1981;68(2):413-421.

26. Bax BE, Bain MD, Fairbanks LD, et al. A 9-yr evaluation of carrier erythrocyte encapsulated adenosine deaminase (ADA) therapy in a patient with adult-type ADA deficiency. Eur J Haematol. 2007;79(4):338-348.

27. Bory C, Boulieu R, Souillet G, et al. Comparison of red cell transfusion and polyethylene glycol-modified adenosine deaminase therapy in an adenosine deaminase-deficient child: measurement of erythrocyte deoxyadenosine triphosphate as a useful tool. Pediatr Res. 1990;28(2):127-130.

28. Abuchowski A, McCoy JR, Palczuk NC, Van ET, Davis FF. Effect of covalent attachment of polyethylene glycol on immunogenicity and circulating life of bovine liver catalase. J Biol Chem. 1977;252(11): 3582-3586.

29. Davis S, Abuchowski A, Park YK, Davis FF. Alteration of the circulating life and antigenic properties of bovine adenosine deaminase in mice by attachment of polyethylene glycol. Clin Exp Immunol. 1981;46:649-652. 
30. Hershfield MS, Buckley RH, Greenberg ML, et al. Treatment of adenosine deaminase deficiency with polyethylene glycol-modified adenosine deaminase. N Engl J Med. 1987;316(10):589-596.

31. Weinberg K, Hershfield MS, Bastian J, et al. T lymphocyte ontogeny in adenosine deaminase-deficient severe combined immune deficiency after treatment with polyethylene glycol-modified adenosine deaminase. J Clin Invest. 1993;92(2):596-602.

32. Hershfield MS, Chaffee S, Sorensen RU. Enzyme replacement therapy with polyethylene glycol-adenosine deaminase in adenosine deaminase deficiency: overview and case reports of three patients, including two now receiving gene therapy. Pediatr Res. 1993;33(1 Suppl):S42-S47.

33. Hershfield MS. PEG-ADA replacement therapy for adenosine deaminase deficiency: an update after 8.5 years. Clin Immunol Immunopathol. 1995;76(3 Pt 2):S228-S232.

34. Ochs HD, Buckley RH, Kobayashi RH, et al. Antibody responses to bacteriophage phi X174 in patients with adenosine deaminase deficiency. Blood. 1992;80(5):1163-1171.

35. Levy Y, Hershfield MS, Fernandez-Mejia C, et al. Adenosine deaminase deficiency with late onset of recurrent infections: response to treatment with polyethylene glycol-modified adenosine deaminase. J Pediatr. 1988;113(2):312-317.

36. Malacarne F, Benicchi T, Notarangelo LD, et al. Reduced thymic output, increased spontaneous apoptosis and oligoclonal B cells in polyethylene glycol-adenosine deaminase-treated patients. Eur J Immunol. 2005;35(11):3376-3386.

37. Chan B, Wara D, Bastian J, et al. Long-term efficacy of enzyme replacement therapy for adenosine deaminase (ADA)-deficient severe combined immunodeficiency (SCID). Clin Immunol. 2005;117(2):133-143.

38. Thompson LF, Van de Wiele CJ, Laurent AB, et al. Metabolites from apoptotic thymocytes inhibit thymopoiesis in adenosine deaminase-deficient fetal thymic organ cultures. J Clin Invest. 2000;106(9):1149-1157.
39. Husain M, Grunebaum E, Naqvi A, et al. Burkitt's lymphoma in a patient with adenosine deaminase deficiency-severe combined immunodeficiency treated with polyethylene glycol-adenosine deaminase. J Pediatr. 2007;151(1):93-95.

40. Kaufman DA, Hershfield MS, Bocchini JA, et al. Cerebral lymphoma in an adenosine deaminase-deficient patient with severe combined immunodeficiency receiving polyethylene glycol-conjugated adenosine deaminase. Pediatrics. 2005;116(6):e876-e879.

41. Blackburn MR, Volmer JB, Thrasher JL, et al. Metabolic consequences of adenosine deaminase deficiency in mice are associated with defects in alveogenesis, pulmonary inflammation, and airway obstruction. J Exp Med. 2000;192(2):159-170.

42. Blackburn MR, Aldrich M, Volmer JB, et al. The use of enzyme therapy to regulate the metabolic and phenotypic consequences of adenosine deaminase deficiency in mice. Differential impact on pulmonary and immunologic abnormalities. J Biol Chem. 2000;275(41):32114-32121.

43. Marwaha VR, Italia DH, Esper F, Hostoffer RW. Extreme thrombocytosis in response to PEG-ADA: early therapeutic and risk indicator. Clin Pediatr (Phila). 2000;39(3):183-186.

44. Chaffee S, Mary A, Stiehm ER, et al. IgG antibody response to polyethylene glycol-modified adenosine deaminase in patients with adenosine deaminase deficiency. J Clin Invest. 1992;89(5):1643-1651.

45. Blaese RM, Culver KW, Miller AD, et al. T lymphocyte-directed gene therapy for ADA-SCID: initial trial results after 4 years. Science. $1995 ; 270(5235): 475-480$.

46. Aiuti A. Gene therapy for adenosine-deaminase-deficient severe combined immunodeficiency. Best Pract Res Clin Haematol. 2004; 17(3):505-516.
Biologics: Targets \& Therapy

\section{Publish your work in this journal}

Biologics: Targets \& Therapy is an international, peer-reviewed journal focusing on the patho-physiological rationale for and clinical application of Biologic agents in the management of autoimmune diseases, cancers or other pathologies where a molecular target can be identified. This journal is indexed on PubMed Central, CAS,

\section{Dovepress}

EMBase, Scopus and the Elsevier Bibliographic databases. The manuscript management system is completely online and includes a very quick and fair peer-review system, which is all easy to use. Visit http://www.dovepress.com/testimonials.php to read real quotes from published authors. 